\title{
Efeito da terapia LED na disfunção temporomandibular: estudo de caso
}

\author{
Effect of LED therapy on temporomandibular disorder: a case study \\ Davidson Ribeiro Costa ${ }^{1} \bowtie$, David Ribeiro Costa ${ }^{2}$, Diego Rodrigues Pessoa ${ }^{1}$, Leandro Júnio Masuloㄹ, \\ Emília Ângela Lo Schiavo Arisawa ${ }^{1}$, Renata Amadei Nicolau ${ }^{1}$ \\ ${ }^{1}$ Curso de Pós-Graduação em Engenharia Biomédica da Universidade do Vale do Paraíba (UNIVAP). São José dos Campos, SP. \\ 2 Curso de Pós-Graduação em Educação Física da Universidade São Judas Tadeu. São Paulo, SP.
}

\section{RESUMO}

OBJETIVOS: Avaliar o efeito da terapia light emitting diode (LED) na disfunção temporomandibular.

DESCRIÇÃO DO CASO: Uma paciente diagnosticada com disfunção temporomandibular foi submetida a quatros sessões de terapia LED com intervalo de sete dias entre as irradiações. No exame inicial da articulação temporomandibular a paciente apresentava abertura de boca sem dor de $23 \mathrm{~mm}$, abertura máxima de $25 \mathrm{~mm}$ e abertura máxima com auxílio de $27 \mathrm{~mm}$. Após o tratamento houve aumento de $7 \mathrm{~mm}$ na abertura sem dor entre a primeira e a última avaliação enquanto que a abertura máxima de boca e a abertura máxima com auxílio aumentaram 6 mm entre a primeira e a última avaliação. Após 21 dias de tratamento detectou-se redução de $50 \%$ dos sítios dolorosos ao exame de palpação. Após o final do tratamento, a escala visual analógica de dor detectou diminuição da intensidade de dor em ambos os lados, e houve diminuição da média total da intensidade de dor. Pela análise das respostas ao questionário Medical Outcomes Study 36-item Short Form Health Survey (SF-36), dos oito domínios de qualidade de vida avaliados, quatro apresentaram melhora.

CONCLUSÕES: No protocolo empregado neste caso, após a terapia LED houve redução da intensidade da dor e aumento da amplitude de movimentos mandibulares. A remissão dos sinais e sintomas da disfunção temporomandibular resultou em melhora na qualidade de vida da paciente.

DESCRITORES: síndrome da disfunção da articulação temporomandibular; fototerapia; terapia com luz de baixa intensidade.

\section{ABSTRACT}

AIMS: To evaluate the effect of light emitting diode (LED) therapy on temporomandibular disorder.

CASE DESCRIPTION: A woman diagnosed with temporomandibular disorder was subjected to four LED therapy sessions at a seven-day interval. In the initial examination of the temporomandibular joint the patient presented a mouth opening without pain of $23 \mathrm{~mm}$, a maximum opening of $25 \mathrm{~mm}$ and a maximum opening with the aid of $27 \mathrm{~mm}$. After the treatment, there was an increase of $7 \mathrm{~mm}$ in the opening without pain between the first and last evaluation, while the maximum opening of the mouth and the maximum opening with aid increased 6 mm between the first and last evaluation. After 21 days of treatment, a 50\% reduction in painful sites was detected on the palpation examination. After the end of the treatment, the visual analog pain scale detected a decrease in pain intensity on both sides, and there was a decrease in the total mean pain intensity. By analyzing the responses to the Medical Outcomes Study 36-item Short Form Health Survey (SF-36), of the eight quality of life domains evaluated, four showed improvement.

CONCLUSIONS: In this case study, after LED therapy there was reduction in pain intensity and increase of the mandibular range of motion. The resolution of the signs and symptoms of temporomandibular disorder resulted in patient's better quality of life.

KEY WORDS: temporomandibular joint dysfunction syndrome; phototherapy; low-level light therapy. 
Abreviaturas: AMM, amplitude dos movimentos mandibulares; ATM, articulação temporomandibular; DTM, disfunção temporomandibular; EVA, escala visual analógica de dor; LED, Light Emitting Diode (diodo emissor de luz); RDC, Research Diagnostic Criteria for Temporomandibular Disorders (Critérios Diagnósticos de Pesquisa em Disfunção Temporomandibular); SF-36, Medical Outcomes Study 36-item Short Form Health Survey; UNIVAP, Universidade do Vale do Paraíba.

\section{INTRODUÇÃO}

A disfunção temporomandibular (DTM) é definida como um conjunto heterogêneo de distúrbios e anomalias que envolvem a articulação temporomandibular (ATM), os músculos mastigatórios e outras estruturas adjacentes da região orofacial. Essa disfunção é a causa mais comum de dor na região facial, depois da de origem dental [1,2]. A prevalência de indivíduos com necessidade de tratamento é de 5-12\% em populações aleatórias [3].

A DTM pode ser classificada em três categorias com base nos sintomas e/ou na sua origem: miogênica, caracterizada por sintomas dolorosos nos músculo da face, que é a forma mais comum; articular, provocada por distúrbios da ATM, podendo estar associada ao deslocamento do disco articular ou a algum processo inflamatório na região articular; degenerativa, podendo estar vinculada a artrite reumatoide ou osteoartrite da ATM [4].

A DTM é caracterizada pela presença de sintomas dolorosos, sendo que o mais relatado pelos pacientes é a dor nos músculos mastigatórios e na região da ATM. Cefaleia, distúrbios otológicos (zumbidos e ouvido entupido) e vertigem também podem estar presentes [4, 5]. Durante o exame clínico dos pacientes com DTM é possível constatar ruídos articulares (estalos e crepitações), aumento do tônus da musculatura mastigatória, sensibilidade da musculatura da face, desvios durante a movimentação mandibular e limitação da amplitude dos movimentos mandibulares (AMM). Esse conjunto de sinais e sintomas podem comprometer atividades diárias como a fala, a mastigação, a deglutição, o bocejo e até mesmo o sono, diminuindo a qualidade de vida dos pacientes com DTM $[5,6]$.

O processo álgico e inflamatório na DTM ocorre por uma série de eventos encadeados por estímulos provenientes dos músculos, vasos sanguíneos, cápsulas articulares e ligamentos. Nesses processos há liberação de mediadores químicos algiogênicos, que podem ativar os receptores nociceptivos. Entre esses mediadores estão os fosfolipídios, que se originam da metabolização do ácido araquidônico, formando a prostaglandina E. No processo inflamatório há a liberação de leucotrienos, e também pode ocorrer destruição de plaquetas, liberando serotonina e determinando edema local. O espasmo muscular, que acompanha localmente a lesão, é mediado por um padrão neural no qual os impulsos nociceptivos, que passam pelos gânglios da raiz dorsal da medula espinhal, são enviados pelas conexões neuronais às células do corno anterior, resultando em contração muscular [3].

O tratamento para DTM é um desafio, visto que a sua etiologia é considerada multifatorial e complexa. São inúmeros fatores que podem contribuir para o surgimento, progressão e perpetuação dessa disfunção. Frequentemente são prescritos fármacos para o relaxamento muscular ou para o alivio dos quadros álgicos. No entanto, o uso de fármacos é indicado em quadros agudos, enquanto na dor crônica, mais frequente na DTM, são necessários métodos não invasivos, devido à extensão do tempo de tratamento [2].

Dentre as alternativas terapêuticas para o tratamento da DTM, encontram-se a terapia com laser de baixa intensidade e a terapia com diodo emissor de luz (light emitting diode - LED) de baixa intensidade, esta última também conhecida como fototerapia. A utilização da terapia a laser de baixa intensidade na região do infravermelho tem sido amplamente divulgada nos meios científicos e clínicos, devido aos efeitos positivos na redução da sintomatologia dolorosa e no aumento da AMM dos pacientes com DTM $[6,7]$. Estudos têm apontado para possível semelhança entre os efeitos da LED em relação aos obtidos com laser [4, 8]. No entanto, os efeitos da terapia LED na DTM ainda não foram amplamente estudados. Portanto, este estudo de caso teve como objetivo avaliar a eficácia da terapia LED no tratamento da DTM.

\section{RELATO DO CASO}

Uma mulher de 21 anos apresentou-se ao Centro de Laserterapia e Fotobiologia (CELAFO) do Instituto de Pesquisa e Desenvolvimento (IP\&D) da Universidade do Vale do Paraíba (UNIVAP), com dor severa na face e trismo, que ocasionava a abertura reduzida da boca. Os sintomas penduravam por um período superior a seis meses. Após avaliação, foi diagnosticada com DTM de origem articular e muscular.

O protocolo de tratamento e a publicação deste caso foram aprovados pelo Comitê de Ética em Pesquisa com Seres Humanos da UNIVAP (no 1.132.239/2015). 
Todos os procedimentos realizados atenderam aos padrões éticos do comitê de pesquisa institucional e à declaração de Helsinque de 1964 e suas emendas posteriores. O consentimento livre e esclarecido e a autorização para divulgação dos dados (incluindo as fotos) foram obtidos da paciente e todos os seus direitos foram protegidos.

A paciente foi submetida a quatros sessões de terapia LED com intervalo de sete dias entre as irradiações. Durante o tratamento a paciente foi orientada a não fazer uso de analgésicos, anti-inflamatórios ou realizar qualquer tipo de tratamento fisioterápico.

\section{Avaliações}

Para as avaliações foram empregados os critérios diagnósticos de pesquisa para DTM (Research Diagnostic Criteria - RDC). Primeiramente foi aplicado o Eixo 2 desse sistema, que consiste em um questionário, classificando os indivíduos com base no grau de dor crônica, depressão e sintomas físicos. A aplicação do questionário foi realizada em sala climatizada, bem iluminada e sem restrição de tempo. Em seguida foi realizado o exame clínico (Eixo 1) que consiste em uma avaliação que aborda a mensuração da AMM e sinais e sintomas relacionados à DTM.

Foram realizadas três avaliações, sendo a primeira antes do início do tratamento, a segunda 15 dias após a primeira irradiação e a última logo após o término do tratamento (21 dias após a primeira irradiação). As três avaliações foram realizadas por um cirurgiãodentista e contemplavam anamnese, exame clínico com mensuração da AMM (aplicação dos eixos I e II dos RDC) e aplicação da escala visual analógica de dor (EVA), bem como a aplicação do questionário de qualidade de vida Medical Outcomes Study 36-item Short Form Health Survey (SF-36) antes e após o tratamento. Nas três avaliações foram seguidas as diretrizes da Associação Internacional de Pesquisa Odontológica, que recomendam uma única medida para cada movimento $[6,9]$, também com base nos RDC para DTM.

$\mathrm{Na}$ avaliação da abertura de boca a paciente foi instruída abrir a boca, sem que houvesse nenhum tipo de desconforto. Na abertura máxima de boca, a paciente foi instruída a abrir a boca o máximo possível. $\mathrm{Na}$ abertura máxima com auxilio, o avaliador posicionou o dedo polegar nos incisivos centrais superiores do voluntário, o dedo indicador em direção aos incisivos centrais inferiores do indivíduo. Nesta posição o avaliador exerceu a ação de alavanca necessária para forçar a abertura de boca da paciente, empregando pressão moderada, que cessou ao comando da paciente $[2,10]$. Para mensuração da abertura de boca foi utilizada uma régua de plástico transparente de $30 \mathrm{~cm}$ de comprimento (Acrilex ${ }^{\circledR}$ ). Foi considerada a distância entre a borda incisal dos incisivos centrais superiores e a dos incisivos inferiores.

A avaliação dos quadros álgicos foi realizada pelo exame clínico (palpação muscular e articular) também com base nos RDC para DTM. Para o registro da dor a EVA foi impressa em uma folha de sulfite (tamanho A4). A EVA consiste em uma escala numérica horizontal de $10 \mathrm{~cm}$, na qual o paciente é instruído a registrar a intensidade da dor através de uma marca com lápis (sobre o valor). A escala de classificação vai de 0 (ausência de dor) a 10 (pior dor imaginável). A EVA é recomenda pela American Dental Association, sendo amplamente utilizada como um critério subjetivo para a avaliação da intensidade da dor [11, 12].

A palpação muscular/articular (RDC - Eixo 1) foi empregada para avaliar a sensibilidade e a dor, seguindo as recomendações descritas por Dworkin e LeResche [13]. A palpação abrangeu as seguintes áreas da região orofacial: músculo temporal em sua porção posterior (área de trás das orelhas até acima das orelhas), porção média (região na depressão em torno de $4-5 \mathrm{~cm}$ lateralmente à borda lateral da sobrancelha) e porção anterior (fossa infratemporal, imediatamente acima do processo zigomático); músculo masseter em sua origem (1 cm à frente da ATM, abaixo do arco zigomático e anteriormente até a borda do músculo), no seu corpo (abaixo do processo zigomático na borda anterior do músculo), na inserção ( $1 \mathrm{~cm}$ superior e anterior ao ângulo da mandíbula); região posterior da mandíbula (entre a inserção do músculo esternocleidomastoideo e a borda posterior da mandíbula); região submandibular (abaixo da mandíbula, $2 \mathrm{~cm}$ anteriormente ao seu ângulo); polo lateral (anterior ao tragus da orelha e sobre a ATM); região do ligamento posterior (meato externo esquerdo).

$\mathrm{Na}$ palpação intraoral foi avaliada a área do músculo pterigoideo lateral, sendo a paciente instruída a abrir a boca e mover a mandíbula para o lado que está sendo examinado. $\mathrm{O}$ dedo indicador do avaliador foi posicionado na porção lateral do rebordo alveolar acima dos molares superiores. O dedo do avaliador foi movido distalmente, para cima, e medialmente para a palpação. Após a palpação do músculo pterigoideo lateral, o dedo do avaliador foi rotacionado para próximo do processo coronoide para a palpação do tendão do temporal.

O questionário SF-36, traduzido e validado para a língua portuguesa [14] foi empregado antes de iniciar 
o tratamento e logo após o término do mesmo. O SF-36 é um questionário auto aplicável de avaliação genérica, que abrange a qualidade de vida [15-17]. Esse instrumento é formado por 36 questões, divididas em oito domínios: capacidade funcional, aspectos físicos, dor, estado geral de saúde, vitalidade, aspectos sociais, aspectos emocionais e saúde mental. Para a pontuação dos domínios é realizado o cálculo da Raw Scale, que é obtido pela diferença entre o valor das questões e o limite inferior multiplicada por 100 e dividida pela a variação. Os valores de cada questão, de limite inferior e variação (Score Range) são fixos e já são estipulados pelo questionário. A Raw Scale varia de zero a 100 , onde zero é o pior e 100 é o melhor para cada domínio [18].

\section{Protocolo de tratamento}

Para a terapia LED foi empregado o equipamento Fisioled $^{\circledR}$ (MMOptics, São Carlos, SP, Brasil) registro ANVISA 80051420003, comprimento de onda $880 \pm$ $20 \mathrm{~nm}$, com modo de emissão contínua e aplicação pontual/contato. Foram empregados 70 segundos/ponto, densidade de energia de $7 \mathrm{~J} / \mathrm{cm}^{2}$ e potência de $0,03 \mathrm{~W}$. Os parâmetros da irradiação eletromagnética foram baseados em estudos prévios [4, 19, 20].

A irradiação foi feita de forma pontual, em contato e perpendicularmente, sobre quatro pontos em forma de cruz na região pré-auricular (Figura 1A), um ponto no meato acústico externo [5-7,20] (Figura 1B), seis pontos nos músculos masseter (Figura 1C), e dois pontos no músculo temporal (feixe anterior) $[2,5,9,20]$.
Os pontos da terapia LED foram demarcados com o auxílio de gabarito plastificado e caneta esferográfica preta.

\section{Resultados das avaliações}

No exame inicial de AMM foi observada uma abertura de boca sem dor de $23 \mathrm{~mm}$, abertura máxima de $25 \mathrm{~mm}$ e abertura máxima com auxílio de $27 \mathrm{~mm}$. A análise da AMM na segunda e na terceira avaliações em relação à inicial mostrou respectivamente: aumento na abertura de boca sem dor, da abertura máxima de boca e aumento da abertura máxima de boca com auxílio. Na abertura sem dor houve aumento de $7 \mathrm{~mm}$ entre a primeira e a última avaliação enquanto que na abertura máxima de boca e na abertura máxima com auxílio o aumento foi de $6 \mathrm{~mm}$ entre a primeira e a última avaliação (Tabela 1).

Tabela 1. Análise das três avaliações da abertura de boca, em uma paciente com disfunção temporomandibular submetida a quatros sessões semanais de terapia com light emitting diode (LED), com intervalo de sete dias entre as irradiações.

\begin{tabular}{ccccccc}
\hline Avaliações & ASD & $\uparrow(\%)$ & AM & $\uparrow(\%)$ & AMA & $\uparrow(\%)$ \\
$\mathbf{1}$ & 23 & - & 25 & - & 27 & - \\
$\mathbf{2}$ & 25 & $6^{*}$ & 28 & $12^{*}$ & 30 & $11^{*}$ \\
$\mathbf{3}$ & 30 & $20^{+}$ & 31 & $10^{+}$ & 33 & $10^{+}$ \\
\hline
\end{tabular}

ASD: Abertura de boca sem dor; AM: Abertura Máxima de boca; AMA: Abertura máxima de boca com auxílio; $\uparrow(\%)$ : Melhora da amplitude em porcentagem;

* Em relação à primeira avaliação; † Em relação à segunda avaliação.

1: avaliação antes do início do tratamento; 2: avaliação 15 dias após a primeira irradiação; 3: avaliação 21 dias após a primeira irradiação.
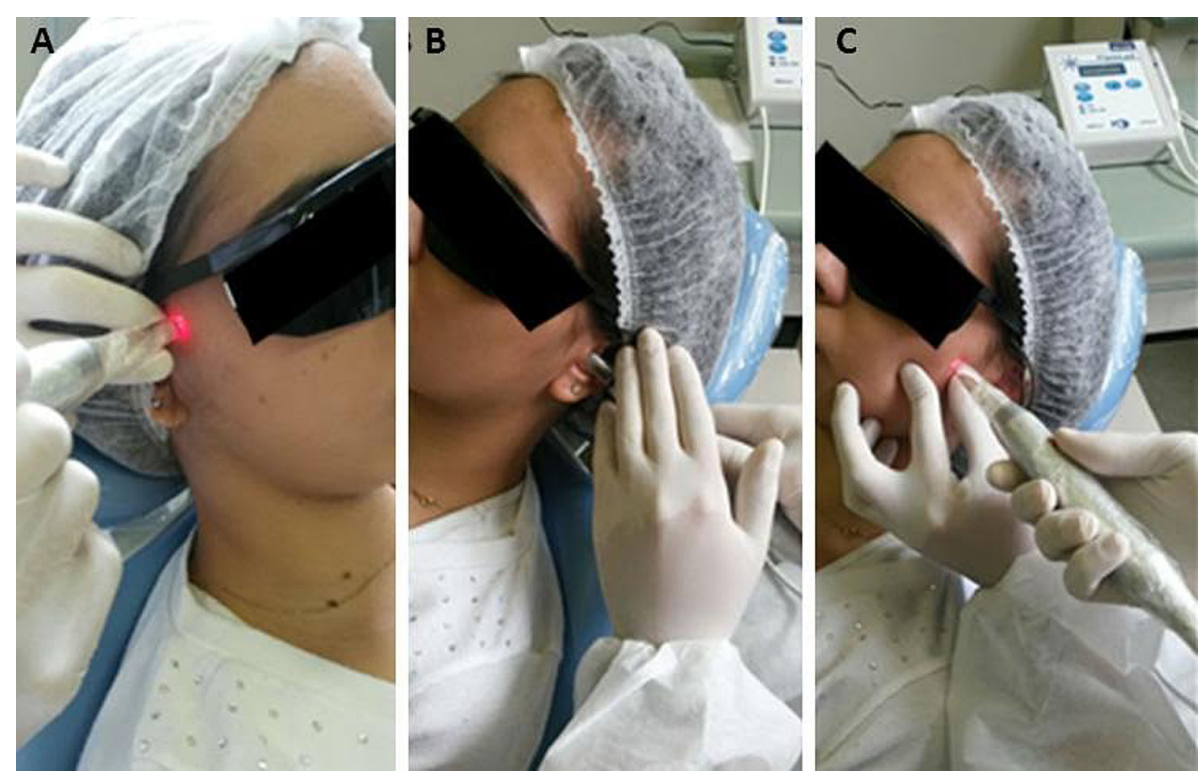

Figura 1. Pontos de aplicação da terapia com light emitting diode (LED) na região orofacial, em paciente com disfunção temporomandibular. (A) região pré-auricular; (B) meato acústico externo; (C) músculo masseter. 
No exame de palpação muscular/articular a paciente relatava dor nos seguintes sítios da região orofacial: músculo temporal (posterior, médio e anterior), músculo masseter (origem, corpo e inserção), região posterior da mandíbula (região dos músculos estilohioideo e digástrico posterior), região submandibular (região dos músculos pterigoideo medial, suprahioideos e digástrico anterior), polo lateral (palpação articular, ligamento posterior), área do pterigoideo lateral (palpação intraoral) e no tendão do temporal (palpação intraoral) de ambos os lados, totalizando 24 sítios. Mediante a EVA, a média total foi de 6,3 na intensidade de dor.

Foram avaliados 24 sítios no exame de palpação muscular e articular, e nas duas primeiras avaliações houve relato de dor pela paciente em todos os sítios avaliados. Após 21 dias detectou-se redução de 50\% dos sítios dolorosos no exame de palpação. Após o final do tratamento foi possível verificar a diminuição da intensidade de dor por meio da EVA em ambos os lados e a diminuição da média total da intensidade de dor. Além disso, o lado direito apresentou a maior média $(7,3)$ na avaliação inicial e as menores médias $(3$ e 0,33$)$ durante as duas últimas avaliações (Figura 2).

Pela análise das respostas ao questionário de qualidade de vida SF-36, dos oito domínios avaliados, $50 \%$ apresentaram melhora. Apresentaram melhora os domínios dor, estado geral de saúde, vitalidade e saúde mental, enquanto nos demais domínios não houve mudança entre as resposta assinaladas antes e após a terapia LED. No domínio da dor verificou-se melhora de $38 \%$, na escala Raw, após o tratamento. Já nos domínios estado geral de saúde, vitalidade e saúde mental, observou-se melhora de $15 \%, 5 \%$ e $8 \%$, respectivamente (Tabela 2 ).

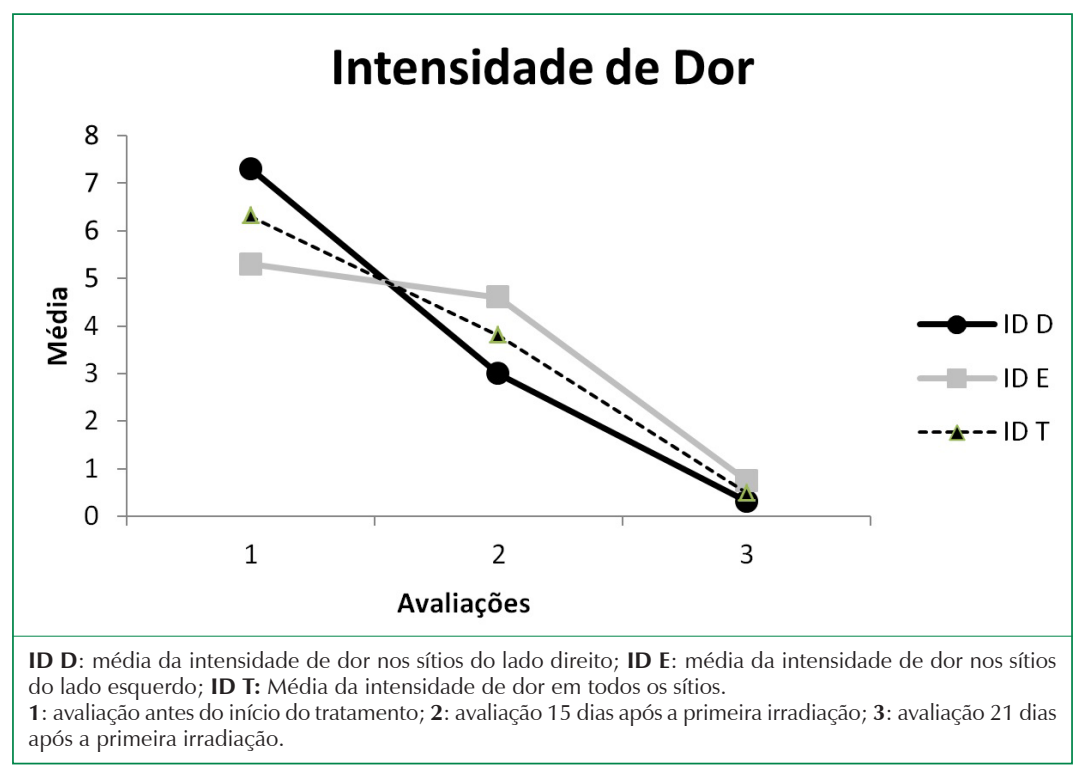

Figura 2. Intensidade de dor por meio da Escala Visual Analógica durante as três avaliações, em uma paciente com disfunção temporomandibular submetida a quatros sessões semanais de terapia com light emitting diode (LED).

Tabela 2. Análise da qualidade de vida por meio do questionário Medical Outcomes Study 36-item Short Form Health Survey (SF-36) antes e após quatro sessões semanais de terapia com light emitting diode (LED) em uma paciente com disfunção temporomandibular.

\begin{tabular}{ccccccccc}
\hline Avaliações & $\begin{array}{c}\text { CF } \\
(\%)\end{array}$ & $\begin{array}{c}\text { LF } \\
(\%)\end{array}$ & $\begin{array}{c}\text { Dor } \\
(\%)\end{array}$ & $\begin{array}{c}\text { ES } \\
(\%)\end{array}$ & $\begin{array}{c}\text { VT } \\
(\%)\end{array}$ & $\begin{array}{c}\text { AS } \\
(\%)\end{array}$ & $\begin{array}{c}\text { AE } \\
(\%)\end{array}$ & $\begin{array}{c}\text { SM } \\
(\%)\end{array}$ \\
\hline Inicial & 85 & 100 & 46 & 47 & 50 & 62,5 & 100 & 60 \\
\hline Final & 85 & 100 & 84 & 62 & 55 & 62,5 & 100 & 68 \\
\hline$\uparrow(\%)$ & 0 & 0 & 38 & 15 & 5 & 0 & 0 & 8 \\
\hline
\end{tabular}

CF: capacidade funcional; LF: limitação por aspectos físicos; ES: estado geral de saúde; VT: vitalidade; AS: aspectos sociais; AE: aspectos emocionais SM: saúde mental; Inicial: avaliação antes do início do tratamento; Final: avaliação logo após o término do tratamento; $\uparrow(\%)$ : porcentagem de melhora dos domínios após a terapia LED. 


\section{DISCUSSÃO}

Estudos com LED são de grande importância na área da saúde, pois esses equipamentos apresentam custo inferior quando comparados ao laser [21]. Segundo Lizarelli et al. [8] o LED com parâmetros apropriados pode produzir efeitos equivalentes ao do laser, justificando estudos na área. Atualmente a terapia a laser de baixa intensidade é amplamente empregada no tratamento da DTM. No entanto, apenas Panhoca et al. [4] realizaram estudo empregando a terapia LED, obtendo resultados positivos no tratamento de sinais e sintomas da DTM.

No presente caso foram obtidos resultados positivos na redução dos pontos dolorosos e da intensidade da dor, no aumento da AMM e na qualidade de vida da paciente submetida ao tratamento. No estudo de revisão de Costa et al. [1], no qual foram avaliados 20 estudos que empregaram a terapia LED no tratamento da DTM, com total de 781 pacientes, também foram observadas redução da dor nos locais de irradiação (músculos mastigatórios e ATM) e diminuição da dor nos locais sensíveis à palpação muscular em $100 \%$ dos estudos. Resultados positivos também foram relatados para a análise de AMM, que apresentou aumento em $90 \%$. A diminuição no número dos pontos dolorosos, da intensidade da dor e o aumento da AMM observados no presente estudo e na literatura podem ser atribuídos aos efeitos promovidos pela terapia LED.

Dentre os principais efeitos da terapia LED encontram-se aumento na síntese de adenosina trifosfato, da microcirculação periférica, da vascularização, do suporte de nutrientes, do fluxo linfático e o favorecimento do funcionamento da bomba de sódio e potássio [22, 23]. Esses efeitos resultam em uma ação anti-inflamatória e analgésica $[4,11,20]$, ocasionando melhora na qualidade de vida dos pacientes com DTM.
No presente caso foi empregada a terapia LED na região do infravermelho, que possui maior capacidade de penetração e absorção, o que possibilita maior efeito fotoelétrico [6]. Esse efeito contribui para a manutenção do potencial de membrana celular, interrompendo a propagação dos estímulos dolorosos aos centros nervosos [24].

Os resultados obtidos neste caso podem ser considerados de grande relevância clínica, pois sem o correto tratamento a DTM pode levar à diminuição das funções orais básicas, como mastigação, deglutição e até mesmo a fala, o que resulta em drástica diminuição na qualidade de vida dos pacientes. De acordo com o protocolo utilizado neste estudo de caso, após a terapia LED houve remissão dos sinais e sintomas da DTM, observando-se efeitos analgésico, anti-inflamatório e biomodulador, resultando em aumento da qualidade de vida da paciente. Contudo, a terapia LED na DTM articular não possui efeito na etiologia ou na causa do distúrbio, sendo uma terapia de suporte para a redução dos sinais e sintomas. Sendo assim, a identificação e a eliminação dos fatores etiológicos são de extrema importância para o sucesso do tratamento em longo prazo.

\section{NOTAS}

Agradecimentos

Os autores Costa DR e Pessoa DR agradecem à Coordenação de Aperfeiçoamento de Pessoal de Nível Superior (CAPES), pelas bolsas de doutorado e mestrado respectivamente.

\section{Apoio financeiro}

Bolsas concedidas pela Coordenação de Aperfeiçoamento de Pessoal de Nível Superior (CAPES).

Declaração de conflito de interesses

Os autores declaram não haver conflitos de interesses relevantes ao conteúdo deste estudo, informam ter tido acesso a todos os dados obtidos e assumem completa responsabilidade pela integridade dos resultados.

\section{REFERÊNCIAS}

1. Costa DR, Ribeiro-Costa D, Delpasso CA, Prianti BM, Masulo LJ, Seefeldt VB, Nicolau RA. Fototerapia de baixa intensidade no tratamento de desordens temporomandibulares: métodos de avaliação e aspectos atuais. XV Encontro Latino-americano de Pós- Graduação - Universidade do Vale do Paraíba: 2015 22,23 Oct; São José dos Campos, SP, Brazil: UNIVAP; 2015.

2. Demirkol N, Sari F, Bulbul M, Demirkol M, Simsek I, Usumez A. Effectiveness of occlusal splints and low-level laser therapy on myofascial pain. Lasers Med Sci. 2014:30(3):1007-12. https://doi.org/10.1007/s10103-014-1522-7

3. Oliveira W. Disfunções Temporomandibulares. Volume único. São Paulo: Artes Médicas, 2002.

4. Panhoca VH, Lizarelli RZ, Nunez SC, Pizzo RA, Grecco C, Paolollo FR, Bagnato VS. Comparative clinical study of light analgesic effecton temporomandibular disorder (TMD) using red and infrared led therapy F. Lasers Med Sci. 2015:30(2):815-22. https://doi.org/10.1007/s10103-013-1444-9 
5. Rodrigues JH, Marques MM, Biasotto-gonzalez DA, Moreira MS, Bussadori SK, Mesquita-ferrari RA, Martins MD. Evaluation of pain, jaw movements, and psychosocial factors in elderly individuals with temporomandibular disorder under laser phototherapy. Lasers Med Sci. 2015:30(3):953-9. https://doi.org/10.1007/s10103-013-1514-Z

6. Pereira TS, Flecha OD, Guimarães RC, Oliveira D, Botelho AM, Glória JCR, Tavano KA. Efficacy of red and infrared lasers in treatment of temporomandibular disorder - a double blind, randomized, parallel clinical trial. Cranio. 2014:32(1): 51-56. https://doi.org/10.1179/0886963413Z.0000000005

7. Catão MCV, Oliveira OS, Costa RO, Carneiro VSM. Avaliação da eficácia do laser de baixa intensidade no tratamento das disfunções temporo-mandibular: estudo clínico randomizado. Rev CEFAC. 2013:15(6):1601-08. https://doi.org/10.1590/ S1516-18462013005000052

8. Lizarelli RFZ, Miguel FAC, Freitas-Pontes KM, Villa GEP, Nunez SC, Bagnato VS. Dentin hypersensitivity clinical study comparing LILT and LEDT keeping the same irradiation parameters. Med Phys. 2010:7(11):805-11. https://doi. org/10.1002/lapl.201010060

9. Godoy CH, Silva PF, Araujo DS, Motta LJ, Biasotto-Gonzalez DA, Politti F, Mesquita-ferrari RA, Fernandes KP, Albertini R, Bussadori SK. Evaluation of effect of low-level laser therapy on adolescents with temporomandibular disorder: study protocol for a randomized controlled trial. Trials. 2012:14(229). https://doi.org/10.1186/1745-6215-14-229

10. Ahrari F, Madani AS, Ghafouri ZS, Tunér J. The efficacy of low-level laser therapy for the treatment of myogenous temporomandibular joint disorder. Lasers Med Sci. 2014:29(2):551-7. https://doi.org/10.1007/s10103-012-1253-6

11. Gökçen-röhlig B, KipirdI S, Baca E, Keskin H, Sato S. Evaluation of orofacial function in temporomandibular disorder patients after low-level laser therapy. Acta Odontol Scand. 2013:71(5):1112-7. https://doi.org/10.3109/00016357.2012. 749517

12. Carvalho CM, Lacerda JÁ, Santos neto FP, Cangussu MC, Marques AM, Pinheiro AL. Wavelength effect in temporomandibular joint pain: a clinical experience. Lasers Med Sci. 2010:25(2):229-32. https://doi.org/10.1007/s10103009-0695-y

13. Dworkin SF, LeResche L. Research diagnostic criteria for temporomandibular disorders: review, criteria, examinations and specifications, critique. Cranio. 1992:6:339-342.

14. Ciconelli RM, Ferraz MB, Santos W, Meinão I, Quaresma M. Tradução para a língua portuguesa e validação do questionário genérico de avaliação de qualidade de vida SF-36 (Brasil SF-36). Rev Bras de Reumatol. 1999:39(3): 143-50.

15. Ruaro JA, Ruaro MB, Frez AR, Daniel CR, Nicolau RA. Qualidade de vida em mulheres com fibromialgia submetidas à laserterapia de baixa intensidade. R Bras Qual Vida. 2014:6(1):46-52. https://doi.org/10.3895/S2175-08582014000100006

16. Ruckenstuhl P, Gerwin AB, Sadoghi P, Glehr M, Holzer LA, Leithner A, Wolf M, Gruber G. Quality of life after volar locked plating: a 10-year follow-up study of patients with intra-articular distal radius fractures. BMC Musculoskelet Disord. 2014:15(250). https://doi.org/10.1186/1471-2474-15-250

17. Villalonga LR, Viniegras CR, Seucjo AH, Leonard DP, Diaz LC, Migues MB. Linfedema y calidad de vida. Rev Cuba Angiol Cir Vasc. 2012:13(1).

18. Pimenta FA, Simil FF, Tôrres HO, Amaral CF, Rezende CF, Coelho TO, Rezende NA. Avaliação da qualidade de vida de aposentados com a utilização do questionário SF-36. Rev Assoc Med Bras. 2008:54(1): 55-60. https://doi.org/10.1590/ S0104-42302008000100021

19. Frare JC, Nicolau RA. Análise clínica do efeito da fotobiomodulação laser (GaAs - 904 nm) sobre a disfunção temporomandibular. Rev Bras Fisioter. 2008:12(1):37-42. https://doi.org/10.1590/S1413-35552008000100008

20. Dostalová T, Hlinakova P, Kasparova M, Rehacek A, Vavrickova L, Navrátil L. Effectiveness of physiotherapy and GaAlAs laser in the management of temporomandibular joint disorders. Photomed Laser Surg. 2012:30(5):275-80. https://doi.org/10.1089/pho.2011.3171

21. Ferreira CLB, Nicolau RA, Oliveira MA, Costa DR, Prianti Júnior ACG. Efeito da terapia LED $(\lambda=945 \pm 20 \mathrm{~nm})$ de baixa intensidade sobre tecido epitelial de ratos diabéticos em processo de reparo. Rev Bras Eng Bioméd. 2013:29(4):404-13.

22. Giuliani A, Lorenzini L, Gallamini M, Massell A, Giardino L, Calzà L. Low infrared laser light irradiation on cultured neural cells: effects on mitochondria and cell viability after oxidative stress. Complementary and Alternative Medicine. 2009:9(8):1-10.

23. Piva JAAC, Abreu EMC, Silva VS, Nicolau RA. Ação da terapia com laser de baixa potência nas fases iniciais do reparo tecidual: princípios básicos. Arq Bras Dermatol. 2011:86(5):947-54. https://doi.org/10.1590/S0365-05962011000500013

24. Ricci R. Estudo in vitro da bioestimulação de células endoteliais em resposta a diferentes dosimetrias do laser de semicondutor fosfeto de índio-gálio-alumínio [dissertation]. [São José dos Campos]: Instituto de Pesquisa \& Desenvolvimento, Universidade do Vale do Paraíba; 2003. 109p. $€$ 